Crime, Histoire \& Sociétés / Crime, History \& Societies

Vol. 21, $n^{\circ} 2$ | 2017

L'histoire de la criminalité et de la justice pénale : propositions de recherche pour le $21^{\mathrm{e}}$ siècle

\title{
Vingt ans de Crime, Histoire \& Sociétés/Crime, History \& Societies
}

Reflets d'un champ de recherche dynamique

Falk Bretschneider, Anja Johansen, René Lévy et Xavier Rousseaux

\section{OpenEdition \\ Journals}

Édition électronique

URL : http://journals.openedition.org/chs/1750

DOI : $10.4000 /$ chs. 1750

ISSN : 1663-4837

Éditeur

Librairie Droz

Édition imprimée

Date de publication : 31 décembre 2017

Pagination : 7-16

ISSN : 1422-0857

Référence électronique

Falk Bretschneider, Anja Johansen, René Lévy et Xavier Rousseaux, « Vingt ans de Crime, Histoire \& Sociétés/Crime, History \& Societies ", Crime, Histoire \& Sociétés / Crime, History \& Societies [En ligne], Vol. 21, $\mathrm{n}^{\circ} 2$ | 2017, mis en ligne le 19 juillet 2020, consulté le 12 janvier 2021. URL : http:// journals.openedition.org/chs/1750; DOI : https://doi.org/10.4000/chs.1750 


\title{
Vingt ans de Crime, Histoire \& Sociétés/ Crime, History \& Societies
}

\author{
Reflets d'un champ de recherche dynamique
}

\section{Falk Bretschneider, Anja Johansen, René Lévy, Xavier Rousseaux}

T a revue Crime, Histoire \& Sociétés/Crime, History \& Societies célèbre cette année

Lson $20^{\mathrm{e}}$ anniversaire. Vingt ans, c'est déjà un âge respectable que beaucoup de revues n'atteignent pas et nous le célébrons en publiant ce numéro spécial consacré à l'avenir de la recherche dans le domaine de l'histoire de la justice pénale et de la criminalité.

\section{LA NAISSANCE DE CHS}

La création d'une revue vise en général à combler un manque et à créer un débouché éditorial pour un champ de recherche émergent. C'est toujours une entreprise collective, engageant un noyau dur de chercheurs qui se connaissent bien et ont souvent noué des liens amicaux. Dans notre cas, la revue est d'abord une émanation de l'International Association for the History of Crime and Criminal Justice (IAHCCJ), un réseau informel de spécialistes de l'histoire de la criminalité et de la justice pénale né en 1978, dans le giron de la Fondation de la Maison des sciences de l'homme (FMSH, Paris) et sous l'impulsion du regretté Herman Diederiks et de Pieter Spierenburg, associés à Michelle Perrot, Nicole et Yves Castan, André Zysberg, Jacques-Guy Petit, Clive Emsley, Jim Sharpe et d'autres. Ce groupe initial s'est rapidement élargi et s'est réuni régulièrement, une ou deux fois par an pour des journées d'études organisées à la FMSH, accompagnées par la publication d'un bulletin annuel.

C'est au sein de ce groupe élargi qu'au tout début des années 1990 a mûri l'idée de créer une revue spécialisée, à mesure que le bulletin annuel tendait à s'étoffer. Le premier projet concret date de 1994, mais le premier numéro n'est paru qu'en 1997.

Nous avions été frappés en effet par le fait qu'en dépit du développement considérable de ce champ de recherche depuis la fin des années 1970, il n'existait qu'une revue annuelle de parution assez irrégulière, Criminal Justice History, animée par Louis Knafla (université de Calgary) et qui a depuis cessé de paraitre. Notre ambition était de produire non pas une revue qui fût le porte-drapeau d'une école particulière, même si nous nous inscrivions naturellement dans une perspective constructiviste de la criminalité et des institutions pénales, mais une revue susceptible d'accueillir les travaux de l'ensemble des spécialistes de l'histoire pénale, sans exclusive. Aujourd'hui encore, CHS est la seule revue internationale d'histoire sociale dans ce domaine, à côté de revues plus régionales ou plus spécialisées.

Cette entreprise n'aurait pu voir le jour sans le soutien indéfectible de la FMSH, et tout particulièrement de son responsable de l'époque, Maurice Aymard, et de 
Max Engammare, le directeur de la Librairie Droz (Genève) qui a d'emblée relevé le défi de publier une revue bilingue. Elle n'aurait pu se poursuivre sans le soutien matériel d'autres institutions - le Centre national du livre (France), la Fondation Herman Bianchi (Pays-Bas), ou plus durablement, le CNRS (France) - et sans le dévouement de tous ceux, trop nombreux pour être nommés ici, qui, au cours de ces années, ont participé au comité de rédaction et au comité scientifique de la revue, sans oublier les secrétaires de rédaction successives, Bessie Leconte et Martine Aubry.

Pour commémorer ces vingt ans, la rédaction a choisi d'interroger un certain nombre de chercheurs, soit à partir d'un regard rétrospectif sur les travaux produits dans leur domaine de spécialité, soit à partir d'une vision prospective sur des champs de recherche à venir. Notre invitation à prospective n'a pas obtenu une réponse de tous les collègues contactés. Les trente-et-une contributions reçues ne représentent donc pas la totalité des champs de recherche dans le domaine qui est le nôtre, et elles ne peuvent pas prétendre à en donner une vision exhaustive, construite et organisée. C'est pourquoi, nous avons ajouté en fin de cette introduction une ouverture bibliographique pour les périodes et domaines non abordés dans ce numéro.

Ce numéro spécial se veut par conséquent une exploration nécessairement incomplète sur la diversité des approches, des terrains et des méthodes. Les textes constituent une approche polyphonique des enjeux, rétrospectifs et prospectifs, de l'histoire du crime, élaborés à partir de l'enracinement de chaque auteur dans son terrain et nourris par une bibliographie choisie.

Quatre orientations fortes se dégagent. Le futur de l'histoire du crime passe par une réflexion croissante sur les processus de globalisation. Il nécessite une interrogation sur les cadres spatiaux et temporels mis en œuvre par les historiennes et historiens. Enfin, il s'inscrit dans un dialogue constant visant à désenclaver les spécialités disciplinaires en sciences humaines et sociales, au profit d'une approche multipolaire des phénomènes criminels et de la réponse des sociétés au crime.

Par conséquent, les contributions de ce numéro spécial ont été classées dans quatre grandes parties thématiques, tout en apportant souvent des éléments de réponse à des problèmes soulevés dans plusieurs d'entre elles.

\section{GLOBALISATIONS}

Thème dominant l'histoire en ce début de XXI ${ }^{e}$ siècle ${ }^{1}$, la globalisation concerne au premier chef l'histoire criminelle. La plupart des contributions à ce volume évoquent le concept. Sans entrer dans les débats sémantiques et épistémologiques ${ }^{2}$, on peut relever que le phénomène de globalisation peut s'entendre de manière géographique, chronologique, disciplinaire ou thématique.

Depuis le développement de ce champ de recherche dans les années 1960, au départ de travaux portant sur l'histoire des sociétés préindustrielles occidentales (XIV $-\mathrm{XVIII}{ }^{\mathrm{e}} \mathrm{s}$.), principalement en Amérique du Nord et en Europe occidentale, l'histoire du crime s'est étendue à de nombreuses sociétés humaines de la planète 3 .

Bayly (2004), Osterhammel (2014)

Voir à ce sujet le numéro thématique de la Revue d'histoire moderne et contemporaine (Coll. 2007).

Voir le point «Temporalités» de cette introduction. 
Dans leur ouverture spatio-temporelle, les chercheurs ont dialogué et intégré de manière croissante les perspectives de nombreuses sciences sociales, voire naturelles (anthropologie biologique, archéosciences, neurosciences) dans l'analyse du crime et de la justice, comme le soulignent les contributions de J. Adler, M.Porret, P. Spierenburg et J.C. Wood ${ }^{4}$. Enfin, la globalisation a touché les méthodologies envisagées et les thématiques abordées. Outre l'approche comparée des modèles, des normes, des pratiques, des représentations dans diverses communautés, sociétés ou États, l'histoire croisée ou enchevêtrée s'est intéressée aux circulations entre sociétés différentes (comme le montre le texte de B. Godfrey).

Les travaux sur la police et sur les pénalités constituent de bons exemples. L'histoire des polices s'écrit désormais dans une perspective globalisante (voir la contribution d'A. Johansen). Les textes de N.Picard et de S. Hynd mettent en relief les circulations des modèles en matière d'usage de la peine capitale ou de châtiments corporels et celui de J. Claustre, E. Lusset, I. Heulland-Donat, en matière d'enfermements (voir aussi Dikötter, Brown (2007). Ces perspectives d'histoire connectée ont renforcé la démarche comparative dans trois domaines. L'étude des sociétés en guerre ou en occupation militaire (voir la contribution de C.Emsley), l'approche croisée des régimes autoritaires et dictatoriaux par T. Marques,T. Ribeiro et l'histoire des entreprises coloniales menée dans les textes de S. Hynd, E. Blanchard, M. Finnane ou D. Fyson. Ainsi les recherches s'intéressent aux pratiques coloniales à l'intérieur d'un Empire colonisateur (britannique, français, espagnol, portugais, italien, néerlandais...), développent la comparaison entre empires coloniaux en Afrique, Amérique, Asie ou encore favorisent l'approche connectée de la «question criminelle » en contexte métropolitain, colonial, ou post colonial, comme le soulignent S. Hynd, E. Blanchard, ou A. Johansen.

En outre, devenue connectée et globale, l'histoire permet d'élargir la recherche à des thèmes jusqu'alors abordés d'un point de vue unilatéral - souvent national. En matière de phénomènes, M. De Koster et H. Reinke soulignent la construction socio-juridique des crimes comme la crimmigration tandis que P. Knepper fait de même pour les crimes internationaux ou transnationaux (piraterie, trafics d'êtres humains, cybercrime...). En matière de réponses sociales au crime, les travaux se multiplient sur les réseaux internationaux normatifs, policiers, judiciaires, ou encore sur la globalisation des représentations du crime et de la justice ${ }^{5}$. Enfin, comme l'évoque $\mathrm{O}$. Rovetta à partir du cas rwandais, un des thèmes dominant au $\mathrm{XX}^{\mathrm{e}}$ siècle est l'émergence d'une justice internationale, liée aux conséquences dramatiques des conflits mondialisés.

Cette globalisation suscite de nombreuses réflexions critiques sur les modalités de construction de ces phénomènes globaux, comme sur leurs niveaux d'échelle, ou les articulations avec les configurations locales, régionales ou nationales. Un intérêt épistémologique de cette approche globalisante est la mise en question d'un point aveugle du discours historien, rappelé par P. Knepper: la «chronocentricité» ou tendance à croire que sa propre génération, ou pour l'historien la période dont il est spécialiste, est un moment pivotal de l'histoire.

Néanmoins cette «globalisation» est elle-même victime de son succès. La généralisation de l'anglais académique comme langue de communication globale

Voir le point «Transdisciplinarités» de cette introduction.

5 Lewis (2014), Shelley (2014), Bruinsma (2015), Newman (2017). 
accélère aussi les possibilités de comparaison au prix d'une distorsion avec les cultures criminelles et judiciaires locales et affaiblit la visibilité de travaux publiés dans des langues scientifiquement dominées ou des sociétés plurilingues. Pour la transmission du savoir historique, de nombreuses communautés nationales ne disposent pas de synthèse à l'échelle de leur nation. Certaines périodisations historiques restent privilégiées, en fonction de leur place dans la construction identitaire nationale. Ainsi $\mathrm{X}$. Rousseaux rappelle combien les travaux sur la domination occidentale du XVI ${ }^{\mathrm{e}}$ au $\mathrm{XX}^{\mathrm{e}}$ siècle continuent à donner une visibilité croissante aux régions conquises par les Empires occidentaux.

La globalisation modifie également les pratiques des chercheurs. Évoluant dans un cadre de plus en plus international, ceux-ci sont tentés de privilégier les objets, les acteurs et les documents transnationaux ou internationaux, plus prometteurs pour leur carrière, au détriment d'études minutieuses au plan local et croisant des sources denses, complexes et variées sur les acteurs, les pratiques ou les représentations collectives ${ }^{6}$.

\section{TERRITOIRES}

Dans cette perspective globalisante, la question des territoires demeure centrale pour l'observation et l'interprétation.

Une des tâches futures de l'histoire du crime sera de bâtir une approche à des niveaux d'échelle à la fois comparables dans le temps et dans l'espace et reflétant la complexité des relations de pouvoir. Dans ce volume les contributions portant sur les aires géographiques ont mis en évidence la difficulté de couvrir des territoires homogènes sur une longue durée. De nombreuses taches blanches demeurent sur la carte criminelle et judiciaire du globe. Certaines aires culturelles ou époques sont absentes de cet aperçu. Relevons ici la remarque de S. Hynd à propos de l'histoire de l'Afrique qu'il importe de dégager de l'héritage de la punitivité coloniale ou du rôle, trop souvent inconsciemment assigné, de miroir d'une altérité archaïque de société sans État. Cette remarque invite les chercheurs à renforcer la comparaison équilibrée entre aires géographiques, qu'elles aient été influencées ou non par la domination européenne.

Si les sociétés issues de l'expansion de l'Europe (occidentale) occupent une large place dans l'historiographie fondée, comme le rappelle X. Rousseaux, sur des travaux nombreux et portant sur une très longue durée, d'autres aires géographiques apparaissent à travers ces bilans, grâce à N. Kollman, sur cinq siècles d'Empire russe, ou à T. Buoye sur l'espace chinois du XVI ${ }^{\mathrm{e}}$ au $\mathrm{XIX}^{\mathrm{e}}$ siècle.

En termes territoriaux, selon les cultures nationales ou l'état du développement de la recherche, les travaux tendront à privilégier un cadre local, régional/provincial, ou étatique. Ainsi, les travaux sur la Russie et la Chine reposent sur des sources essentiellement produites par l'État impérial dans son entreprise de concentration du pouvoir, alors que les travaux canadiens relevés par D. Fyson s'expriment davantage dans un cadre provincial (Québec, Ontario...) ou «ethnique»: «nations» anglaises, françaises ou autochtones. Quant à l'espace européen, comme le rappelle

6 Guldi et Armitage (2015). 
$\mathrm{X}$. Rousseaux, il pose à la fois la question d'une identité récente (la construction politique de l'Union européenne) et d'une identification millénaire.

L'émergence des empires entraîne une reconfiguration de la notion de territoire. Le cas le mieux documenté est celui de l'expansion britannique, en Afrique, en Asie ou en Amérique du Nord grâce aux contributions comparables de D.Fyson, S. Hynd ou M.Finnane. La question du territoire est, comme l'écrit M. Finnane, liée au «momentous clash of imperial power and settler colonialism with indigenous cultures». Dans ces perspectives, la gestion des territoires autochtones ou les relations entre les politiques des métropoles colonisatrices, des pouvoirs coloniaux et des minorités ethniques constituent le creuset d'une véritable histoire globale. Pour sortir de la domination historiographique des empires européens, des travaux similaires sur d'autres configurations impériales (russe/soviétique, chinoise, arabe, indienne, ottomane, japonaise...) seraient bienvenus dans les années à venir.

Le contraste entre les régions rurales et les grandes agglomérations urbaines constitue une autre ligne de partage pour l'histoire du crime, soulignée par l'histoire coloniale. Pour les $\mathrm{XVIII}{ }^{\mathrm{e}}-\mathrm{XX}{ }^{\mathrm{e}}$ siècles particulièrement, la recherche s'est focalisée sur les métropoles: Calcutta, Sidney, Montréal et les grandes villes africaines, alors que les territoires ruraux sont négligés. Or ceux-ci présentent des spécificités, dont la moindre n'est pas la présence très faible d'un pouvoir central, métropolitain ou colonial, soulignée par E. Blanchard. Ici encore, les travaux «coloniaux» éclairent la construction de l'État dans les métropoles colonisatrices sur une période plus longue. Sur une longue durée millénaire, la sécurisation du territoire rural représente un défi pour le cœur géographique et politique des États modernes, dans leur lutte pour l'hégémonie (Russie, États-nations européens, Chine).

Mouvante et variable, la notion de territoire doit s'articuler avec celle de frontière. Rappelons ici l'analyse de longue durée de Charles Tilly qui appuie le développement de l'État moderne en Europe sur un double mouvement: la création de territoires pacifiés en interne et le transfert des violences vers des frontières extérieures ${ }^{7}$. L'embrigadement des détenteurs de la force (la noblesse) au service d'armées souveraines constitue le moteur de cette création d'espaces pacifiés.

La création des «frontières nationales» prend un sens nouveau, dans le cadre de la conquête coloniale comme de l'occupation militaire. Les frontières ne sont alors pas uniquement géographiques mais, comme le relève E. Blanchard, raciales ou culturelles. Dans ce cadre conquérant, la notion d'empire de différences ${ }^{8}$ ou de centre et périphéries, suggérée par N. Kollman, permet de relativiser le modèle hégémonique de l'État moderne, en observant comment peut se construire une idéologie impériale, dans la tolérance forcée ou voulue d'une diversité de pratiques communautaires de gestion du crime.

Enfin, la gestion autoritaire des peuples par des régimes isolationnistes met en évidence leurs effets sur les sensibilités aux circulations des populations. Comme le soulignent M.De Koster et H. Reinke, les conséquences politico-militaires de la guerre froide (rideau de fer) pèsent aujourd'hui sur les représentations opposées des migrations chez les Européens de l'Est et de l'Ouest.

Tilly (1992).

8 Barkey (2006). 


\section{TEMPORALITÉS}

Depuis leurs débuts, les recherches dans le champ de l'histoire de la criminalité et de la justice pénale ont connu des priorités chronologiques différentes selon les historiographies nationales. Si en France et en Angleterre, les deux pays où ce domaine s'est développé le plus largement, les recherches couvrent une longue période allant du Moyen Âge à l'époque contemporaine, le XIX ${ }^{\mathrm{e}}$ siècle reste pourtant la période de prédilection de nombreux travaux. En revanche, en Allemagne, mais aussi dans certaines historiographies de l'Europe de l'Est (par exemple en Tchéquie), l'époque moderne est toujours largement prédominante, alors que les études concernant les $\mathrm{XIX}^{\mathrm{e}}$ et $\mathrm{XX}^{\mathrm{e}}$ siècles restent rares (exception faite de l'histoire de la criminologie et de celle des techniques policières où l'époque contemporaine est naturellement bien plus présente). En revanche, la période médiévale avant 1300, l'Antiquité (surtout autre que romaine) et, a fortiori, la préhistoire restent toujours, et partout, largement dans l'ombre de l'historiographie. La contribution d'H. Ménard fournit quelques explications à ces lacunes - manque de sources, déficits au niveau des cadres explicatifs (la plupart des grandes approches théoriques s'intéressant peu ou pas du tout à ces époques) - mais indique aussi quelques pistes de recherche jusqu'alors délaissées et concernant notamment une «criminalité du quotidien » qui reste souvent plus facile à saisir pour d'autres époques. Malheureusement, le comité de rédaction n'a pas réussi à convaincre un ou une collègue médiéviste actif dans ce champ de nous dresser un bilan des recherches récentes dans ce domaine un échec que l'on peut lire comme un signe de la faible présence, en comparaison avec d'autres époques, du Moyen Âge dans l'ensemble des travaux portant sur la justice pénale. Cela vaut encore davantage pour l'étude de formes de déviance et de régulation sociale dans des sociétés sans écriture, où l'apport de l'ethnologie reste indispensable (même si la proximité entre historiens et ethnologues, tissée autrefois par l'anthropologie historique, s'est quelque peu distendue ces dernières années).

Le point de vue d'un médiéviste permet pourtant de donner davantage de poids à un aspect assez négligé dans l'un des domaines les plus étudiés: celui de la peine de mort. Souvent abordée à partir d'approches plus générales - comme le concept de «processus de civilisation» forgé par Élias - la mise en œuvre de la peine capitale revêtait également, voire prioritairement, des significations religieuses dans des sociétés profondément marquées par le poids de la religion et de l'Église. Comme le montre P. Schuster dans sa contribution, l'échafaud est devenu, à la fin du Moyen Âge, le lieu d'une véritable mise en scène de la sacralité. Cette influence de la religion sur les pratiques pénales se confirme au moment de la Réforme quand, d'une part, le nombre des crimes punis par cette sanction augmente considérablement et, d'autre part, l'usage de l'épée devient de plus en plus la règle lors des exécutions - du moins dans les pays germaniques où l'impact des controverses théologiques semble être plus important qu'ailleurs (par exemple en France où la décapitation reste jusqu'à la fin de l'Ancien Régime un privilège de la noblesse). Ces interrogations peuvent également être lues comme le signe d'une ouverture du champ entier vers des questionnements davantage animés par des approches d'histoire culturelle (dans la diversité que revêt ce terme dans les différentes historiographies nationales). Ceux-ci se reflètent aussi dans les contributions de K. Härter et M.Porret qui dressent un premier bilan des recherches dans des domaines particulièrement dynamiques au cours de ces dernières années: les rapports entre criminalité et diversité culturelle (ce qui inclut un intérêt 
pour l'impact des minorités et des phénomènes migratoires dans le pénal) ainsi que les représentations populaires du crime et de la justice pénale qui se sont formées dans la sphère publique, en donnant une place grandissante au topos de la sécurité qui sert à son tour au développement d'un appareil juridico-médical destiné à identifier et punir le coupable.

Si l'orientation culturelle d'un grand nombre de recherches récentes est commune à des travaux consacrés à différentes époques, sans pour autant proposer de véritables perspectives transpériodiques, un domaine en particulier se distingue par une approche faisant du dépassement du cloisonnement chronologique sa marque de fabrique: les travaux sur l'histoire de l'enfermement. Longtemps focalisés, dans le sillage des études d'obédience foucaldienne, sur la prison pénale du XIX ${ }^{\mathrm{e}}$ s., ils se sont ces dernières années résolument ouverts aux époques moderne et médiévale, en adoptant en outre une perspective comparative tournée vers d'autres «milieux clos », par exemple monastères, asiles ou hôpitaux, en remettant au goût du jour une idée déjà développée par la sociologie des années 1960 (notamment dans les travaux d'E. Goffman). Comme l'indiquent J. Claustre, I. Heullant-Donat et E. Lusset, cette approche - qui s'intéresse à une histoire des pratiques d'enfermement inscrite dans la longue durée - tente donc de désenclaver un domaine dont les racines sont bien plus anciennes que ce que l'historiographie a longtemps voulu faire croire.

\section{TRANSDISCIPLINARITÉS}

L'histoire de la criminalité et de la justice pénale s'est toujours conçue ellemême comme faisant partie d'un champ disciplinaire plus large, incluant notamment l'histoire du droit et la sociologie, mais aussi la criminologie. Ses thèmes, approches et orientations théoriques impliquaient par conséquent une perspective résolument interdisciplinaire telle qu'elle s'exprime également dans les engagements pris par notre revue. Cette interdisciplinarité traditionnelle fait aujourd'hui place à une plus grande variété d'ouvertures disciplinaires. En témoigne par exemple l'intérêt pour l'espace de la justice pénale, esquissé dans la contribution de F. Bretschneider, et qui se nourrit d'une coopération parfois ancienne entre historiens et géographes (comme en France), mais davantage encore des réorientations thématiques dans un spectre plus large de sciences sociales et humaines incluant la philosophie et l'anthropologie. La perspective spatiale permet non seulement de poser de nouvelles questions (par exemple celle de la participation de la justice pénale à la spatialisation du pouvoir politique), mais aussi d'apporter de nouveaux résultats dans des domaines établis, par exemple l'étude de la violence juvénile dans des contextes urbains - comme le montre le texte de K. Weinhauer qui souligne l'inscription de cette violence dans des processus de communication reliés eux-mêmes à des pratiques spatiales se nourrissant d'imaginaires individuels et collectifs. En relation avec les nouvelles approches spatiales de l'histoire du crime et de la justice, l'intérêt pour une histoire matérielle et visuelle du crime - telle qu'elle est présentée par X. Rousseaux s'inspire davantage de travaux d'archéologues ou d'historiens de l'art, mais aussi du poids grandissant que prennent les humanités numériques dans ce domaine. Comme le montre T. Hitchcock dans sa contribution, les nouvelles technologies ne permettent pas seulement au chercheur d'obtenir un accès plus facile à certains types de sources (même si c'est souvent par le biais d'offres commerciales); elles lancent aussi un 
défi à un certain «conservatisme méthodologique» régnant actuellement dans notre champ. Apprendre à faire un usage raisonnable et raisonné des nouvelles possibilités engendrées par la numérisation massive de certains matériaux est une tâche qui reste largement à accomplir dans les prochaines années.

Un deuxième domaine d'approfondissement de coopérations interdisciplinaires concerne les sciences cognitives, disciplines en pleine effervescence, et qui permettent de poser à nouveaux frais certaines vieilles questions. C'est le cas du problème des taux d'homicides et de leur évolution historique, objet de controverses régulièrement rejouées, et qui pourrait profiter d'une plus large ouverture disciplinaire, comme le montre le texte de J.S. Adler. Celui de J.C. Wood plaide en faveur d'une intégration accrue des résultats de la psychologie évolutive dans les recherches sur l'histoire de la violence, en soulignant qu'une telle approche peut aider à trouver une perspective théorique commune et à mieux comprendre «le sens» de la violence. Si ces propositions ne feront certainement pas l'unanimité des historiens engagés dans ce champ, elles montrent du moins le potentiel d'irritation productive que représente tout regard au-delà des frontières disciplinaires. Enfin, trois contributions - celles de R. Wetzell, D. Churchill et R. Roth - donnent à voir les rapports féconds entre histoire et criminologie. Ceux-ci sont tout d'abord perceptibles dans le domaine de l'histoire de la criminologie d'abord, qui s'ouvre vers d'autres champs historiques (notamment l'histoire de l'État-providence) et évolue vers une histoire transnationale des sciences. On les repère aussi dans les échanges entre histoire et criminologie, qui font fructifier des interrogations et orientations méthodologiques et théoriques communes. Ils s'expriment enfin dans une coopération renforcée entre ces deux disciplines, un dialogue qui en s'ancrant désormais dans une tradition ancienne continuera à donner une profondeur historique à des interrogations souvent limitées à la seule actualité.

Signalons par ailleurs que d' autres réorientations et coopérations transdisciplinaires n'ont pu être évoquées dans ce numéro spécial de notre revue faute, le plus souvent, d'avoir pu trouver une ou un auteur(e) prêt(e) à nous fournir un bref bilan dans ces domaines. C'est le cas pour l'histoire des émotions, thématique centrale notamment pour l'évolution historique de la violence ou des crimes passionnels, mais aussi pour les représentations de la criminalité dans l'espace public et l'attitude des pouvoirs publics. Autre exemple : l'étude de l'influence des développements technologiques sur l'histoire du crime et de la justice pénale - quels changements engendrent l'avènement de la photographie pour le travail criminologique, l'apparition de l'automobile pour l'action policière, la naissance de l'ordinateur pour les formes de criminalité et leur poursuite, etc. ? De même, nous aurions aimé qu'un texte approfondisse les rapports entre notre domaine et celui de l'histoire du droit, qui a fait ces dernières années des progrès considérables notamment pour l'étude du fonctionnement des cours et tribunaux. Il aurait été également judicieux de disposer d'un point de vue plus détaillé sur le renouveau des travaux sociologiques sur la prison, qui sont animés par une actualité brûlante où se mêlent discriminations sociales et ethniques, défaillances criantes des pouvoirs publics et formes extrêmement violentes de contestation comme le terrorisme. Enfin, le tour d'horizon proposé à nos lectrices et lecteurs fait également largement l'impasse sur les médias et leur impact sur les représentations individuelles et collectives du crime: romans et télévision (où fleurit le genre du polar), cinéma et séries (souvent consacrées à des thématiques historiques touchant également le domaine la justice pénale, que ce soit dans Borgia, The Tudors ou Babylon Berlin), mais aussi nouveaux médias (comme les jeux vidéo, souvent eux aussi à caractère historique). 


\section{REMARQUE HISTORIOGRAPHIQUE}

À propos des périodes et aires négligées par les contributions, le lecteur pourra relever l'existence d'une abondante littérature scientifique sur l'histoire criminelle, souvent récente. Un rapide aperçu de la bibliographie met en évidence des recherches sur le crime sous des intitulés classiques: droit, crime, châtiments, ou branchés sur des thématiques récentes issues de l'histoire criminelle: banditisme, crime organisé, violence, crimes sexuels, genre, contrôle social, répression, policing, True crime..., appliquées aux différentes civilisations du globe.

Ainsi en complément de la contribution d'H. Ménard sur Rome, en est-il des mondes antiques du Moyen-Orient et de la Méditerranée 9 . Il en va de même pour les mondes arabes ou l'Empire ottoman ${ }^{10}$; pour la péninsule indienne ${ }^{11}$, pour la Chine $e^{12}$, le Japon $^{13}$ et le Sud-Est asiatique ${ }^{14}$. Des recherches sur l'Afrique et l'Amérique avant les colonisations occidentales ${ }^{15}$ complètent les contributions d'E. Blanchard et de S. Hynd dans ce volume. Pour les périodes plus récentes, notons des travaux sur les sociétés latino-américaines ${ }^{16}$ ou les sociétés russe et chinoise, qu'il s'agisse de l'empire tsariste ${ }^{17}$, de l'Union soviétique ${ }^{18}$, de la Chine républicaine ${ }^{19}$ ou de la Chine populaire ${ }^{20}$. Enfin, les chercheurs s'intéressent au crime et à la justice dans les sociétés autochtones ${ }^{21}$.

$9 \quad$ Alston et Hopwood (1999); Bauschatz (2013); Bertrand-Dagenbach et al. (1999, 2005); Cantarella (2000); Cassayre (2010); Coll. (1977); Coll. (1984); Démare-Lafont (1999); Harries (2007); Hunter (1994); Joannès (2000); Kelly (2011); MacDowell (1963); Ménard (2004); Müller-Wollermann (2004); Pavon Torrejon (2003); Rivière (2004); Rollinger et al. (2012); Tetlow (2004, 2005).

10 Barkey (1996); Boğaç (2003); Faroqhi (1995); Gingeras (2014); Lange et Fierro (2009); Lévy-Aksu (2013); Martel-Thoumian (2012); Peirce (2003) ; Peters (2006); Petry (2012); Schull (2014); Semerdjian (2008); White (2011); Zarinebaf (2010).

11 Banerjee (2006); Fischer-Tine et Tambe (2008); Singha (1998).

12 Antony (2003); Dikötter (2002); Hegel (2011).

13 Botsman (2007); Itaru (2012); Kwan-Wai (1975); Murray (1987).

14 Rafael (1999).

15 Bohannan (1967); Dalgleich (2005); Eleechi (2006); Novak (2014).

16 Barreneche (2006); Bretas (1997a et b); Johnson et al. (2013); Salvatore et al. (2001).

17 Ackeret (2007); Adams (2006); Hasegawa (2017); Kaczynska (1994); McReynolds (2013); Nethercott (2012).

18 Barenberg (2014); Clark (1993); Connor (1972); Dobson (2011); Dowling (2017); Feofanov et Barry (1996); Hardy (2016); Healey (2009); Huskey (1986); Ivanova (2000); Khlevniuk (2004); Kowalski (2009); Shelley (1996); Solomon (1996).

19 Dikötter (2002); Ng (2014).

20 Bakken (2005); Liang Lu (2015); Lu Miethe (2010); Mühlhahn (2009); Seymour Anderson (1999); Trevaskes $(2007,2012)$.

21 French (2003); Knafla et Swainger (2006); Nielsen et Silverman (1996, 2009); Thalia (2013). 


\section{LA MISSION DE CRIME, HISTOIRE \& SOCIÉTÉS/ CRIME, HISTORY \& SOCIETIES}

Revue bilingue publiant des articles et des compte-rendus d'ouvrages en français et en anglais, Crime, Histoire \& Sociétés/Crime, History \& Societies a joué le rôle d'une passerelle, encourageant le dialogue entre chercheurs travaillant dans des réseaux linguistiques différents. La mission que s'est assignée notre revue, d'inclure des recherches de toutes les régions du monde, est ambitieuse; elle offre des opportunités mais présente aussi quelques difficultés pratiques. Bien que tous les résumés soient publiés dans les deux langues, certains chercheurs ou institutions sont réticents à s'intéresser à une revue dont une grande partie du contenu n'est pas accessible à l'ensemble des lecteurs. Inversement, les chercheurs capables de lire dans les deux langues profitent largement de l'ouverture intellectuelle et géographique de la revue.

Du fait de ce caractère bilingue, la revue embrasse les traditions universitaires française, britannique et nord-américaine. Son approche éditoriale se caractérise dès lors par une grande souplesse vis-à-vis des conventions de style et de présentation, ce qui la rend particulièrement accueillante pour les chercheurs dont la langue principale n'est ni l'anglais, ni le français et dont les travaux concernent avant tout un public réduit ou non occidental. Dans le monde académique du $21^{\mathrm{e}}$ siècle, les chercheurs sont de plus en plus poussés à s'internationaliser, ce qui implique fréquemment de publier dans une langue autre que leur langue natale. Crime, Histoire \& Sociétés/Crime, History \& Societies se donne pour mission prioritaire d'accueillir les contributions à notre domaine des chercheurs d'autres aires linguistiques, plus réduites ou noneuropéennes.

Une autre mission importante que s'est donnée Crime, Histoire \& Sociétés/ Crime, History \& Societies est de faciliter l'insertion de jeunes chercheurs dans le champ de l'histoire de la criminalité et de la justice pénale, en leur proposant un lieu d'expression où les post-doctorants et les chercheurs en début de carrière peuvent faire leurs premières armes au côté de collègues ayant plusieurs décennies d'expérience dans ce domaine. Dans cet effort pour encourager de jeunes chercheurs talentueux qui proposent des démarches et des perspectives nouvelles, le Prix Herman Diederiks joue un rôle central, avec l'appui de la Fondation de la Maison des Sciences de l'Homme (France). Notre espoir est que la revue puisse aider à consolider les carrières des jeunes chercheurs en publiant des travaux prometteurs, quand bien même leur style serait moins poli que ne l'exigent les normes académiques britanniques ou françaises traditionnelles.

Depuis vingt ans, Crime, Histoire \& Sociétés/Crime, History \& Societies s'est efforcé de rassembler des contributions à l'histoire de la criminalité et de la justice pénale du monde entier et pour toutes les époques. Depuis 1997, la revue a fortement contribué à faire de ce thème, naguère marginal pour les historiens, un domaine reconnu, de sorte qu'à l'avenir on ne pourra plus écrire de synthèses historiques générales sans y inclure des développements sur la criminalité et la justice pénale. Nous espérons que ce numéro spécial contribuera à cette évolution.

[La bibliographie se trouve p. 26-30] 